\title{
PfelKI, a eukaryotic initiation factor $2 \alpha$ kinase of the human malaria parasite Plasmodium falciparum, regulates stress-response to amino-acid starvation
}

\author{
Clare Fennell ${ }^{\dagger 1,2}$, Shalon Babbitt ${ }^{\dagger 3}$, Ilaria Russo ${ }^{3}$, Jonathan Wilkes ${ }^{4}$, \\ Lisa Ranford-Cartwright ${ }^{5}$, Daniel E Goldberg*3 and Christian Doerig*1,6
}

\begin{abstract}
Address: ${ }^{1}$ INSERM U609, Wellcome Centre for Molecular Parasitology, Biomedical Research Centre University of Glasgow, 120 University Place, Glasgow, G12 8TA, UK, 2Institute of Immunology and Infection Reasearch, School of Biological Sciences, University of Edinburgh, West Mains Road, Edinburgh, EH9 3JT, UK, ${ }^{3}$ Departments of Medicine and Molecular Microbiology, Howard Hughes Medical Institute, Washington University Box 8230, 660 S. Euclid Ave, St. Louis, MO 63110, USA, ${ }^{4}$ Wellcome Centre for Molecular Parasitology, University of Glasgow, 120 University Place, Glasgow G12 8TA, UK, ${ }^{5}$ Division of Infection and Immunity, Faculty of Biomedical and Life Sciences, University of Glasgow, 120 University Place, Glasgow G12 8TA, UK and 'INSERM U609, Global Health Institute, Ecole Polytechnique Fédérale de Lausanne (EPFL), Station 19, CH-1015 Lausanne, Switzerland

Email: Clare Fennell - cfennell@staffmail.ed.ac.uk; Shalon Babbitt - sebabbit@artsci.wustl.edu; Ilaria Russo - russo@borcim.wustl.edu; Jonathan Wilkes - jw149j@udcf.gla.ac.uk; Lisa Ranford-Cartwright - L.Ranford-Cartwright@bio.gla.ac.uk; Daniel E Goldberg* - goldberg@borcim.wustl.edu; Christian Doerig* - christian.doerig@epfl.ch

* Corresponding authors †Equal contributors
\end{abstract}

Published: 12 May 2009

Malaria Journal 2009, 8:99 doi:10.1 186/1475-2875-8-99

This article is available from: http://www.malariajournal.com/content/8/1/99

(C) 2009 Fennell et al; licensee BioMed Central Ltd.

This is an Open Access article distributed under the terms of the Creative Commons Attribution License (http://creativecommons.org/licenses/by/2.0), which permits unrestricted use, distribution, and reproduction in any medium, provided the original work is properly cited.
Received: 5 December 2008

Accepted: 12 May 2009

\begin{abstract}
Background: Post-transcriptional control of gene expression is suspected to play an important role in malaria parasites. In yeast and metazoans, part of the stress response is mediated through phosphorylation of eukaryotic translation initiation factor $2 \alpha$ (elF2 $\alpha$ ), which results in the selective translation of mRNAs encoding stress-response proteins.

Methods: The impact of starvation on the phosphorylation state of PfelF2 $\alpha$ was examined. Bioinformatic methods were used to identify plasmodial elF2 $\alpha$ kinases. The activity of one of these, PfelKI, was investigated using recombinant protein with non-physiological substrates and recombinant PfelF2 $\alpha$. Reverse genetic techniques were used to disrupt the $p$ feik $I$ gene.

Results: The data demonstrate that the Plasmodium falciparum elF2 $\alpha$ orthologue is phosphorylated in response to starvation, and provide bioinformatic evidence for the presence of three elF $2 \alpha$ kinases in P. falciparum, only one of which (PfPK4) had been described previously. Evidence is provided that one of the novel elF $2 \alpha$ kinases, PfelKI, is able to phosphorylate the $P$. falciparum elF2 $\alpha$ orthologue in vitro. PfelKI is not required for asexual or sexual development of the parasite, as shown by the ability of pfeik $I^{-}$parasites to develop into sporozoites. However, elF $2 \alpha$ phosphorylation in response to starvation is abolished in pfeik $l$-asexual parasites
\end{abstract}

Conclusion: This study strongly suggests that a mechanism for versatile regulation of translation by several kinases with a similar catalytic domain but distinct regulatory domains, is conserved in $P$. falciparum. 


\section{Background}

Human malaria is caused by infection with intracellular protozoan parasites of the genus Plasmodium that are transmitted by Anopheles mosquitoes. Of four species that infect humans, Plasmodium falciparum is responsible for the most virulent form of the disease. The transition from one stage of the life cycle to the next must be tightly regulated, to ensure proliferation and differentiation occur when and where appropriate; this is undoubtedly linked to differential gene expression. Analysis of the P. falciparum transcriptome during the erythrocytic asexual cycle reveals an ordered cascade of gene expression [1], and the various developmental stages display distinct transcriptomes; how this is orchestrated remains obscure. Initial investigation of the $P$. falciparum genome revealed a paucity of transcriptional regulators [2], although this picture has recently been challenged by the recent identification of the ApiAP2 transcription factor family [3]. There is nevertheless a large body of evidence suggesting that posttranscriptional control is an important means of gene regulation in $P$. falciparum. Examples include the relatively small number of identifiable transcription-associated proteins, abundance of CCCH-type zinc finger proteins commonly involved in modulating mRNA decay and translation rates [2] and translational repression during gametocytogenesis [4-6].

In mammalian cells, regulation of gene expression is a key mechanism in the mediation of stress responses, which may be achieved by influencing transcription or translation. The Stress Activated Protein kinases (SAPKs), specifically JNKs and p38 kinases, are subfamilies of mitogen activated protein kinases (MAPK) that are expressed in most eukaryotic cells, and respond to a variety of stress conditions [7]. Although the parasite kinome includes two MAPK homologues, none of these are members of the SAPK subfamily [8-10]. In contrast, the $P$. falciparum kinome contains a phylogenetic cluster of three kinases with homology to eukaryotic Initiation Factor $2 \alpha$ (eIF2 $\alpha$ kinases, which in other organisms regulate translation in response to stress [10]. Interestingly, the related apicomplexan parasite Toxoplasma gondii has been shown to differentiate from tachyzoites to bradyzoites on exposure to a number of cellular stresses, concomitant with an increase in phosphorylation of TgeIF $2 \alpha$, indicating a possible role for this mechanism in parasite differentiation [11].

Phosphorylation of eukaryotic initiation factor $2 \alpha$ at residue Ser51 in response to stress is a well-characterized mechanism of post-transcriptional control that regulates initiation of translation [12-17]. In mammalian cells this phosphorylation event is mediated by four distinct protein kinases, called the eIF2 $\alpha$ kinases: general control nonderepressible-2 (GCN2), haem-regulated inhibitor kinase
(HRI), RNA-dependent protein kinase (PKR), and PKRlike endoplasmic reticulum kinase (PERK). These enzymes contain a similar catalytic domain allowing them to phosphorylate the same substrate, but have different accessory domains that regulate kinase activation in response to different signals. In GCN2 the functional kinase domain is followed by a histidyl-tRNA synthetase (HisRS)-like domain [18], which is the major motif for sensing amino acid starvation and triggering kinase activation; PERK has a transmembrane domain allowing it to reside in the endoplasmic reticulum membrane; the $\mathrm{N}$ terminal domain can protrude into the lumen of the ER to sense unfolded proteins, while the catalytic domain extends into the cytoplasm where its substrate and effector mechanism lie; human PKR contains an RNA binding domain and responds to viral infection; and HRI contains haem binding sites to modulate translation of globin chains according to the availability of haem. In this way the eIF2 $\alpha$ kinases can integrate diverse stress signals into a common pathway [12-14,19].

Translation initiation requires the assembly of the $80 \mathrm{~S}$ ribosome on the mRNA, which is mediated by proteins known as eukaryotic initiation factors (eIFs). Formation of the 43S pre-initiation complex depends on binding of the ternary complex that consists of the heterotrimeric Gprotein eIF2 ( $\alpha, \beta$ and $\gamma$ subunits), methionyl-initiator tRNA (met-tRNAi) and GTP [13]. Initiation of translation and release of the initiation factors involves hydrolysis of GTP to GDP, which leaves an inactive eIF2-GDP complex. Before further rounds of translation initiation can occur eIF2 must be reactivated by exchange of GDP for GTP [13]. The presence of a phosphate group on the $\alpha$ subunit of eIF2 inhibits recycling of inactive eIF2-GDP to active eIF2-GTP by limiting the activity of the guanine nucleotide exchange factor, eIF2B [20]. The consequence of activity of the eIF $2 \alpha$ kinases therefore is global translation repression, since initiation complexes cannot form. In spite of the generalized reduction in translation, selected mRNAs are translated, whose products shapes the subsequent stress response. Reduced translation conserves energy and nutrients, allowing time for the cell to adapt appropriately to the stress conditions. This mechanism is conserved in the vast majority of eukaryotes. One notable exception is the Microsporidium Encephalitozoon cuniculi, whose kinome does not include eIF $2 \alpha$ kinases (or other stress-response kinases), a probable adaptation to its parasitic lifestyle [21]. It is, therefore, of interest to investigate the extent to which malaria parasites may rely on eIF2 $\alpha$ phosphorylation for stress-response and/or life cycle progression.

A cluster of three sequences that includes PfPK4, a protein kinase that was previously described as a putative eIF2 $\alpha$ kinase [22], was identified in the $P$. falciparum kinome on 
the basis of catalytic domain similarity $[10,23]$. Here, evidence is provided that the P. falciparum eIF $2 \alpha$ orthologue is phosphorylated in response to amino acid starvation. Bioinformatics analysis reveals that $P$. falciparum encodes three eIF2 $\alpha$ kinases, one of which, Plasmodiumfalciparum eukaryotic Initiation Factor Kinase-1 (PfeIK1), is indeed be able to phosphorylate P. falciparum eIF2 $\alpha$ in vitro. Reverse genetics experiments show that inactivation of the pfeik1 gene does not affect asexual growth, gametocytogenesis or further sexual development, since pfeik1- sporozoites can be formed in the mosquito vector; in contrast, pfeik1- parasites are unable to phosphorylate eIF2 $\alpha$ in response to amino-acid starvation.

\section{Methods \\ Bioinformatic analysis}

BLASTP analysis was used to identify the closest human and Plasmodium berghei orthologues of the PfeIF2 $\alpha$ kinases. Catalytic domains of the putative PfeIF $2 \alpha$ kinases as defined by the alignment of $P$. falciparum kinases [10] were aligned with the four human eIF $2 \alpha$ kinases and other $P$. falciparum and human sequences that were selected to represent all kinase subfamilies. The sequences were aligned using the HMMER package against a profile generated from our previous kinome analysis [10]. After removal of gaps and positions with a low quality of alignment, alternate phylogenies generated with the neighbour joining method were visualized using NeighbourNet implemented on SplitsTree version 4 [24].

BLASTP searches of PlasmoDB using metazoan eIF2 $\alpha$ sequences as queries identified PF07_0117 as the P. falciparum homologue of eIF $2 \alpha$, which was then confirmed by reciprocal analysis. Alignment of these sequences was performed using ClustalW.

\section{Molecular cloning}

\section{PfelKI}

A 1278 bp fragment encoding the catalytic domain of PfeIK1 (PF14_0423) was amplified from a P. falciparum cDNA library using the Phusion polymerase (Finnzymes), using the following primers: forward, GGGGGGATCCATGGGGAAAAAAAAACATGG，reverse GGGGGTCGACCGTAAAAAGTACACTTTCGTG. The primers contained BamHI and SalI restriction sites, respectively (underlined). The Taq polymerase (Takara) was used to add adenine tails to enable cloning of the product into the pGEM-T Easy vector (Promega) for sequencing. The correct sequence was removed by digestion with Bam HI and SalI and inserted into the expression vector pGEX-4T3 (Pharmacia). A catalytically inactive mutant was obtained by site directed mutagenesis of Lys 458 to Met using the overlap extension PCR method [25] (forward: CTTATGCATTAATGATTATAAG, reverse: CTTATAATCATTAATGCATAAG).
PfelF $2 \alpha$

Oligonucleotide primers were designed to amplify the complete coding sequence of PfeIF2 $\alpha$ (PF17_0117) by PCR from a cDNA library of the P. falciparum clone 3D7, using the Phusion polymerase (Finnzymes). The primers used were as follows: forward, GGGGGGATCCATGACTGAAATGCGAGTAAAAGC and reverse, GGGGGTCGACTTAATCTTCCTCCTCCTCGTC (restriction sites are underlined). Taq polymerase (Takara) was used to add adenine tails to enable cloning of the 990 bp product into the pGEM-T Easy vector (Promega) for amplification and sequencing. The correct sequence was removed by digestion with BamHI and SalI and inserted into the expression vector pGEX-4T3 (Pharmacia). A mutant of PfeIF2 $\alpha$ designed to be refractory to phosphorylation was obtained by site directed mutagenesis (Ser ${ }^{59}$ - Ala) using the overlap extension PCR technique [25], (primers: forward, CTTATGCATTAATGATTATAAG, reverse, CTTATAATCATTAATGCATAAG).

All inserts were verified by DNA sequencing (The Sequencing Service, Dundee, UK) prior to expression of recombinant proteins or transfection of $P$. falciparum.

\section{Recombinant protein expression}

Expression of recombinant GST fusion proteins was induced in E. coli (strain BL21, codon plus) with $0.25 \mathrm{mM}$ Isopropyl Thiogalactoside (IPTG). After induction, bacteria were grown at $16^{\circ} \mathrm{C}$ overnight and the resulting bacterial pellets were stored at $-20^{\circ} \mathrm{C}$ until use. All subsequent work was done on ice, centrifugation steps at $4{ }^{\circ} \mathrm{C}$. Protein extraction was performed by digestion of bacterial pellets for $5 \mathrm{~min}$. with lysozyme (Sigma), followed by $10 \mathrm{~min}$. in lysis buffer $(1 \times$ PBS, $2 \mathrm{mM}$ ethylenediaminetetraacetic acid (EDTA), $1 \mathrm{mM}$ dithiothreitol (DTT), 0.5\% Triton $\times 100,1 \mathrm{mM}$ Phenyl Methyl Sulphonate (PMSF), Benzamidine Hydrochloride Hydrate (BHH), $1 \times$ complete cocktail protease inhibitors (Roche)). Bacterial lysates were sonicated at $20 \%$ amplitude (Bioblock Scientific, Vibracell 72405$), 5 \times 15 \mathrm{sec}$. pulses/15 sec. rest, and cleared by centrifugation $13000 \mathrm{~g}, 15 \mathrm{~min}$. GST-fusion proteins were purified by incubation of cleared lysates on glutathione agarose beads (Sigma) for 2 hours, followed by four washes with lysis buffer and eluted for $20 \mathrm{~min}$. in elution buffer (Tris $40 \mathrm{mM}, \mathrm{pH} 8.7,75 \mathrm{mM} \mathrm{NaCl}, 15 \mathrm{mM}$ reduced glutathione). Protein concentration was monitored using the Bradford assay (Biorad reagent). Kinase assays were carried out immediately after purification.

\section{Kinase assay}

Kinase reactions $(30 \mu \mathrm{l})$ were carried out in a standard kinase buffer containing $20 \mathrm{mM}$ Tris- $\mathrm{HCl}, \mathrm{pH} 7.5,20 \mathrm{mM}$ $\mathrm{MgCl}_{2}, 2 \mathrm{mM} \mathrm{MnCl}$, phosphatase inhibitors; $10 \mathrm{mM}$ $\mathrm{NaF}, 10 \mathrm{mM} \beta$-glycerophosphate, $10 \mu \mathrm{M}$ ATP and 0.1 MBq $\left[\gamma^{3}{ }^{32} \mathrm{P}\right]$ ATP, using $2 \mu \mathrm{g}$ recombinant kinase, and 10 
$\mu \mathrm{g}$ non-physiological substrate ( $\alpha$-casein, $\beta$-casein), or recombinant GST-PfeIF $2 \alpha$. Reactions were allowed to proceed for $30 \mathrm{~min}$. at $30^{\circ} \mathrm{C}$ and stopped by addition of reducing Laemmli buffer, 3 minutes, $100^{\circ} \mathrm{C}$. Samples were separated by SDS-PAGE and phosphorylation of kinase substrates assessed by autoradiography of the dried gels.

\section{Plasmodium falciparum genetic manipulation}

A gene disruption plasmid was produced for PF14_0423 in the plasmid pCAM-BSD [26] that contains the gene conferring resistance to blasticidin. The oligonucleotide pair GGGGGGATCCGTAATGAAAGTAAAAAATAAG/ GGGGCGCCGGCGAGGTGAAATATAATGAATTGTTCC, containing Bam HI and NotI sites (underlined) was used to amplify a 789 bp fragment for insertion to pCAM-BSD. Ring stage parasites were electroporated with $50-100 \mu \mathrm{g}$ plasmid DNA, as previously described [26]. Blasticidin (Calbiochem) was added to a final concentration of 2.5 $\mu \mathrm{g} / \mathrm{ml} 48$ hours after transfection to select for transformed parasites. Resistant parasites appeared after 3-4 weeks and were maintained under selection. After verification by PCR that $p f e i k 1$ - parasites were present, the population was cloned by limiting dilution in 96 well plates $(0.25 / 0.5 / 1.0$ parasite per well). Genotypic analysis enabled selection of independent $p$ feik 1 - clones for further phenotypic analysis.

\section{Parasite culture and mosquito infection}

Plasmodium falciparum clone 3D7 was cultured as previously described [27]. In brief, asexual cultures were maintained in complete RPMI at a haematocrit of 5\%, between $0.5 \%$ and $10 \%$ parasitaemia. Asexual growth cycle was analyzed by flow cytometric assessment of DNA content as previously described [28]. Gametocytogenesis was induced as described previously [29]; briefly, gametocyte cultures were set up at $0.5-0.7 \%$ parasitaemia in $6 \%$ haematocrit (using human blood not more than 7 days after the bleed), in an initial volume of $15 \mathrm{ml}$ in $75 \mathrm{~cm}^{2}$ flasks. Cultures were maintained for 4-5 days until 8-10\% parasitaemia was reached and parasites appeared stressed after which the volume was increased to $25 \mathrm{ml}$. For each clone a mixture of day 14 and day 17 gametocyte cultures were fed to Anopheles gambiae, through membrane feeders as described [29]. Female mosquitoes were dissected 10 days post-infection and midguts examined by light microscopy for presence of oocysts. Sporozoite invasion of salivary glands was assessed by removal of salivary glands 16 days post-infection and examination by light microscopy. DNA was extracted from oocyst-positive midguts using previously published methods [30]. Fisher's exact test was used to compare infection prevalence between oocyst and sporozoite stages, where appropriate.

\section{Preparation of parasite pellets}

Parasite pellets were obtained by saponin lysis: erythrocytes were centrifuged at $1300 \mathrm{~g}$ for $2 \mathrm{~min}$. at room temperature, washed in an equal volume of Phosphate Buffered Saline (PBS), pH 7.5, and centrifuged at $1300 \mathrm{~g}$ for $2 \mathrm{~min}$. at $4^{\circ} \mathrm{C}$. Erythrocytes were lysed on ice by resuspension and repeated pipetting in $0.15 \%$ saponin in PBS. The PBS volume was then increased and parasites recovered by centrifugation at $5500 \mathrm{~g}$ for $5 \mathrm{~min}$., at $4^{\circ} \mathrm{C}$. After two washes in PBS, the parasite pellets were stored at $80^{\circ} \mathrm{C}$.

\section{Plasmodium falciparum amino acid starvation assay}

Plasmodium falciparum 3D7 parasites and clonal lines of pfeik1- and pfeik2- parasites were synchronized to the late ring stage, cultured in complete RPMI at 2\% haematocrit, and grown to approximately $8-10 \%$ parasitaemia. The parasites were washed two times in $1 \times$ PBS, equally partitioned and washed with either complete RPMI or RPMI medium lacking amino acids, after which, the parasites were re-plated in their respective medium. The plates were incubated at $37^{\circ} \mathrm{C}$ with $5 \% \mathrm{CO}_{2}$ for 5 hours. After 5 hours, one culture maintained in amino acid free medium was supplemented with complete RPMI, and re-incubated at $37^{\circ} \mathrm{C}$ for an additional 45 minutes. Post-incubation, the parasites were isolated by tetanolysin (List Biological) treatment, washed with $1 \times$ PBS buffer containing Complete $^{\mathrm{TM}}$ protease inhibitor cocktail (Roche), $2 \mathrm{mM} \mathrm{NaF}$, and $2 \mathrm{mM} \mathrm{Na}_{3} \mathrm{VO}_{4}$. Samples were resuspended in $2 \times$ SDSLaemmli buffer. Parasite proteins were resolved by SDSPAGE and transferred to nitrocellulose for immunoblotting.

\section{Antibodies and immunoblotting}

Rabbit anti-phosphorylated eIF2 $\alpha$ (Ser 51) was purchased from Cell Signaling Technology (Danvers, MA). Rat antiBiP was acquired from the Malaria Research and Reference Reagent Resource Center (ATCC, Manassas, VA). Secondary antibodies used were conjugated with horseradish peroxidase (HRP). For immunoblotting, nitrocellulose membranes were blocked with $5 \%$ BSA in TBS- $0.1 \%$ Tween 20 (TBST) for 1 hour at room temperature. Rabbit anti-phosphorylated eIF2 $\alpha$ (Ser 51) was diluted 1:1000 in TBST. Rat anti-BiP was diluted 1:10,000 in TBST. Respective secondary antibodies were diluted 1:20,000. Bound antibodies were detected with Western Lightning $^{\mathrm{TM}}$ Chemiluminescence reagent (Perkin Elmer).

\section{Southern blotting}

To obtain genomic DNA, parasite pellets were resuspended in PBS and treated with $150 \mu \mathrm{g} / \mathrm{ml}$ proteinase $\mathrm{K}$ and $2 \%$ SDS at $55^{\circ} \mathrm{C}$ for 4 hours. The DNA was extracted using phenol/chloroform/isoamyl alcohol (25:24:1), and precipitated in ethanol with $0.3 \mathrm{M}$ sodium acetate at $20^{\circ} \mathrm{C}$. Restriction digests were carried out with HindIII. 
Probes were labelled with alkaline phosphatase using the Gene Images AlkPhos Direct Labelling kit (Amersham).

\section{Results and Discussion}

\section{Stress-dependent phosphorylation of the P. falciparum} elF2 $\alpha$ orthologue

BLASTP searches of PlasmoDB using metazoan eIF2 $\alpha$ sequences were used to identify PF07_0117 as the P. falciparum orthologue, which was confirmed by reciprocal analysis. The alignment of $P$. falciparum eIF $2 \alpha$ with sequences from $T$. gondii, human, rice and E. cuniculi is shown in Figure 1A. Overall, the P. falciparum sequence shares $\sim 70 \%$ identity with $T$. gondii eIF $2 \alpha$ and $\sim 50 \%$, $\sim 40 \%$ and $\sim 28 \%$ with the orthologues in humans, rice and E. cuniculi, respectively. Importantly, the serine that is targeted for phosphorylation is conserved in all species. Furthermore, eIF2 $\alpha$ contacts the kinase through a large number of residues that interact with the surface of the kinase domain. These residues are also conserved in most species, as are residues that protect the regulatory serine from the activity of other kinases [31] (Figure 1A); interestingly, several of these are not conserved in the $E$. cuniculi orthologue, which is consistent with the absence of eIF2 $\alpha$ kinases in this organism [21].

The presence of the target serine residues, and of residues which in other species are involved in interaction with eIF2 $\alpha$ kinases, suggests that PfeIF2 $\alpha$ may be regulated by
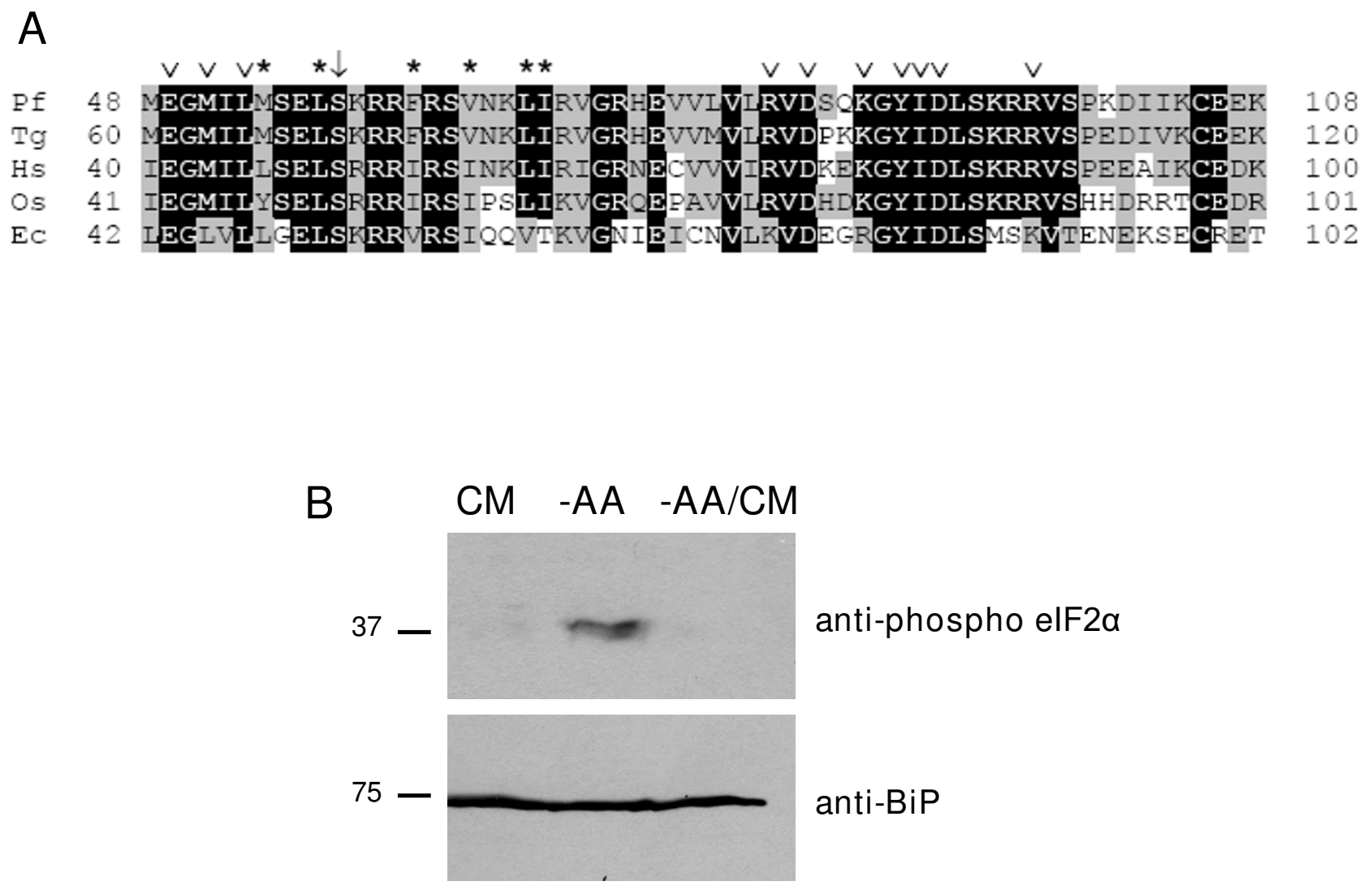

\section{Figure I}

The $\boldsymbol{P}$. falciparum elF $2 \alpha$ orthologue is phosphorylated in response to amino-acid starvation. A: Alignment of PfelF2 $\alpha$ with orthologous sequences from $T$. gondii $(\mathrm{Tg})$, human $(\mathrm{Hs})$, rice $(\mathrm{Os})$ and $E$. cuniculi $(\mathrm{Ec})$. Sequences surrounding the conserved regulatory serine, ( $P$. falciparum numbering: M48 - K 108) are shown. Residues that are identical in all sequences are highlighted in black, residues that are identical or similar are marked in grey. The arrow indicates the serine that is the target of elF2 $\alpha$ kinases. Open arrow heads $(v)$ indicate residues involved in contacting the kinase domain, asterisks $(*)$ indicate conserved residues that protect the phosphorylation site from the activity of other kinases. B. Western blot analysis of PfelF $2 \alpha$ phosphorylation. A 3D7 parasite culture synchronized to the late ring stage was equally partitioned into individual cultures. Growth of the parasites was continued up to 5 hours at $37^{\circ} \mathrm{C}$ in either complete RPMI medium (CM) or in RPMI lacking amino acids (-AA). CM was added back to one amino acid-deprived culture, and re-incubated for an additional 45 minutes. Total lysates from the parasites were prepared for SDS-PAGE, followed by immunoblotting with antibodies against phosphorylated elF2 $\alpha$ (anti-phospho elF2 $\alpha$ ) and the endoplasmic reticulum (ER) marker, BiP (anti-BiP), which served as the loading control. 
phosphorylation under stress conditions. To test this hypothesis, cultured intraerythrocytic parasites were starved of amino acids, and the phosphorylation status of PfeIF2 $\alpha$ was monitored by western blot using an antibody that specifically recognizes the phosphorylated form (Ser51) of human eIF2 $\alpha$, reasoning that the high level of sequence conservation between the human and plasmodial sequences would allow cross-reaction of the antibody (Figure 1B). Indeed, the antibody recognized the expected $37-\mathrm{kDa}$ band in parasite extract, and the intensity of the signal was considerably stronger in the lane containing extracts from parasites that had been stressed by aminoacid starvation than in extracts from unstressed parasites, despite equal quantities of the eIF2 $\alpha$ factor (as quantitated with a non-phosphodependent antibody). Furthermore, this effect was removed by restoring the amino acids in the culture medium. This demonstrates that the $P$. falciparum equivalent residue of human eIF2 $\alpha$ Ser51 is phosphorylated in response to starvation.

\section{Identification of elF2 $\alpha$ kinases in P. falciparum}

Bioinformatics approaches were then used to identify $P$. falciparum protein kinase(s) potentially responsible for this response. An analysis of the complete complement of $P$. falciparum protein kinases [10] identified a distinct phylogenetic cluster of three sequences, PF14_0423, PFA0380w and PFF1370, the latter of which (called PfPK4) had previously been characterized as an eIF2 $\alpha$ kinase [22]. Reciprocal BLASTP analysis using the putative catalytic domains as queries confirmed the homology of these three genes with the eIF2 $\alpha$ kinase family. A Hidden Markov Model (HMM) was used to generate an alignment of the three $P$. falciparum sequences with those of human eIF2 $\alpha$ kinases; sequences of kinases from other families were included as outgroups. The resulting alignment was used to generate a phylogenetic tree (Figure 2A), which clearly shows that the three $P$. falciparum genes cluster with the eIF $2 \alpha$ kinases, as opposed to other kinase families, confirming their relatedness to this family. Interestingly PfeIK1 (PF14_0423), on which the present study focuses, clusters most closely with GCN2, which is suggestive of a role in response to nutrient levels.

The PF14_0423 gene model proposed in PlasmoDB [32] predicts a single intron that falls close to the 5 ' end of the sequence so that the kinase domain is encoded entirely within the second exon. All the residues that are required for catalytic activity [33] are present in the kinase domain, suggesting the gene encodes an active enzyme. The sequence shares the feature of insertions within the catalytic domain with other eIF2 $\alpha$ kinases [34] (Figures 2B and 2C). Three of the human eIF2 $\alpha$ kinases have $\mathrm{N}$-terminal extensions containing regulatory domains; the fourth, GCN2, has extensions on either side of the kinase domain (reviewed in [35]). As PfeIK1 has extensions on both sides of the catalytic domain, it is most similar to GCN2 not only in the sequence of its catalytic domain, as the phylogenetic tree (Figure 2A) demonstrates, but also in overall structure (Figure 1C). Furthermore, the C-terminal extension of PfeIK1 contains an "anti-codon binding" domain (Superfamily entry SSF52954) that may mediate binding to uncharged tRNAs, a function that is performed in GCN2 by the HisRS domain present in the C-terminal extension (Figure 1C) [18]. This adds weight to the possibility that PfeIK1 is involved in the response to amino acid starvation, like GCN2. The other functional domains present in the GCN2 extensions were not recognisable in PfeIK1.

\section{Kinase activity of recombinant PfelKI}

In order to verify that the pfeik1 gene encodes a functional kinase, the catalytic domain was expressed as a GST fusion protein in E. coli. A recombinant protein of the expected size (76 kDa) was obtained and purified for use in kinase assays. The protein appeared as a doublet in most preparations, with both bands reacting with an anti-GST antibody. Kinase assays were performed with $\alpha$ - or $\beta$-casein as substrates, in the presence or absence of GST-PfeIK1 (Figure $3 \mathrm{~A}$ ). A weak signal was detectable with $\beta$-casein on the autoradiogram even in the absence of the kinase, indicating a low level of contaminating kinase activity in the substrate itself. This signal was much stronger in the presence of GST-PfeIK1, and a signal was also observed with $\alpha$ casein, which was not labelled in the absence of the kinase. Furthermore, a signal at a size matching that of the upper band in the GST-PfeIK1 doublet was also seen, indicating possible autophosphorylation, an established property of at least some mammalian eIF2 $\alpha$ kinases, including GCN2 [34,36-38]. GCN2 autophosphorylation occurs on two threonine residues in the activation loop [36], only one of which conserved in PfeIK1 (Figure 2B). Autophosphorylation was more clearly seen in the absence of any exogenous substrate (Figure 3B). The possible functional relevance of PfeIK1 autophosphorylation remains to be determined. Taken together, these data suggest that PfeIK1 possesses catalytic activity. To ensure that the signals were not due to co-purified activities from the bacterial extract, the assays were repeated using a catalytically inactive mutant (Lys458 $\rightarrow$ Met) of GST-PfeIK1. These reactions yielded an identical pattern as the reaction containing no recombinant kinase (Figure 3A), confirming that the phosphorylation of the caseins is due to GSTPfeIFK1, and that the recombinant kinase can autophosphorylate.

In order to establish whether PfeIK1 is an eIF2 $\alpha$ kinase as predicted, its activity was tested towards recombinant $P$. falciparum eIF2 $\alpha$ expressed as a $64 \mathrm{kDa}$ GST fusion. Figure 3B (left lane) shows that GST-PfeIK1 can phosphorylate wild-type GST-PfeIF2 $\alpha$. The signal appears very weak, 
A
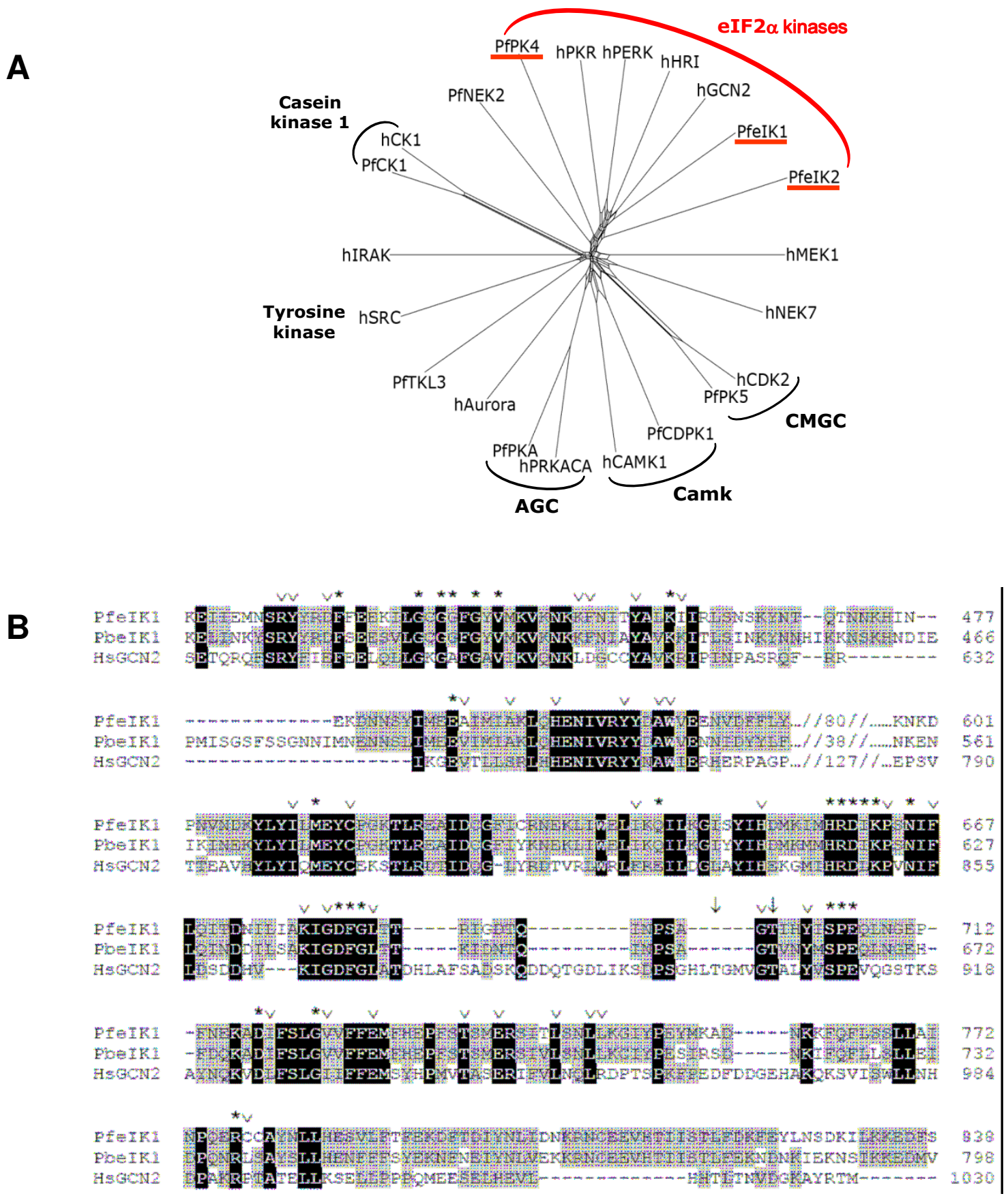

C

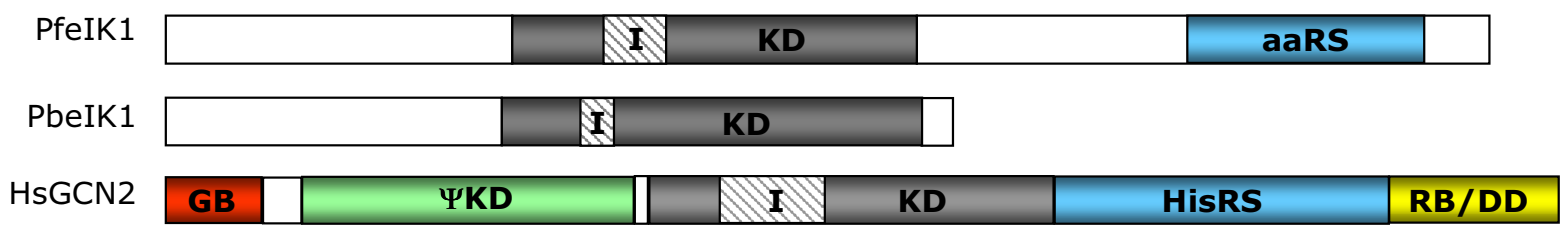

Figure 2 (see legend on next page) 
Figure 2 (see previous page)

Bioinformatic analyses of $\boldsymbol{P}$. falciparum elF2 $\alpha$ kinases. A: Phylogenetic tree showing clustering of PfelF $2 \alpha$ kinases with human elF2 $\alpha$ kinases. Sequences: PfelKI: PFI4_0I I4; PfelK2: PFA0380w; PfPK4: PFFI370; PKR: GI:4506 103; HRI: GI:6580979; PERK: GI:I8203329; GCN2: GI:652877I7; MEKI: GI:400274; IRAKI: GI:68800243; Aurora: GI:37926805; CDK2: GI:I942427; PfCKI: PFII_0377; PfNEK2: Pfel290w; PfPK5: MALI3PI.279; PfPKA: PFII685w; PFTKL3: PFI3_0258; Pfb08I5w: PfCDPKI; hCAMKI: GI:4502553; hPRKACA: GI:46909584; hCSNKId: GI:20544I45; hNEK7: GI:19424I32; hSRC: GI:4885609. B. Alignment of the catalytic domains of PfelKI, PbelKI and human GCN2. Identical residues in all three kinases are in black boxes, residues that are identical in two sequences of the three sequences, or that are similar are boxed in grey. The number of residues comprising the inserts between domains IV and V are marked between //-//. Asterisks (*) mark residues conserved among kinases in general, while open arrowheads $(V)$ indicate residues specifically conserved among elF $2 \alpha$ kinases. The downwards arrow marks the threonine residues that are targets for autophosphorylation in GCN2. PlasmoDB accession numbers: PfelKI: PFI4_0423, PbelKI: PB000582.03.0 GenBank accession number: HsGCN2: GI:652877I7. C: Schematic of the domain structures of PfelKI, PbelKI and GCN2. Kinase domains (KD) are in grey, hatched regions represent the inserts (I) within the kinase domains and regions with no identified function are white. Additional characterized domains of GCN2 are as follows: red; N-terminal GCNI binding domain (GB), green; pseudo-kinase domain ( $\Psi K D)$, blue; histidyl-tRNA synthetase (HisRS), yellow; ribosome binding and dimerisation domain (RB/DD).

which may be explained by the fact that the recombinant kinase contains only the catalytic domain and may not mimic the enzyme in a fully activated, physiological status. Indeed, an activation mechanism for GCN2 has been proposed [37], in which a conformational alteration of the so-called "hinge region" of the catalytic domain is induced by uncharged tRNA binding to the HisRS domain, which would favour productive binding of ATP to the active site. Such a positive effect of the regulatory domain would not be possible with GST-PfeIK1, since it contains only the catalytic domain.

Consistent with the hypothesis that PfeIK1 may regulate translation through PfeIF $2 \alpha$ phosphorylation, mutation of the predicted target for phosphorylation in the substrate (Ser59 $\rightarrow$ Ala) prevents labelling with the recombinant enzyme (Figure 3B).

\section{Generation of pfeik I- clones}

Microarray data available in PlasmoDB $[1,39]$ indicate that pfeik 1 is expressed in asexual parasites; it can be hypothesized that the kinase plays a role in the parasite's stress response, and may therefore (i) not be essential for the asexual cycle, and (ii) be involved in regulation of gametocytogenesis, similar to the function of a eIF2 $\alpha$ kinase in $T$. gondii stage transition from tachyzoite to bradyzoite. P. falciparum clones that do not express PfeIK1 were generated to test these hypotheses. The strategy used to disrupt expression of the kinase relies on single crossover homologous recombination, and has been used successfully to knock-out other $P$. falciparum protein kinase genes $[40,41]$. Briefly, a plasmid based on the PCAM-BSD vector [26] containing a cassette conferring resistance to blasticidin and an insert comprising the central region of the PfeIK1 catalytic domain, was transferred by electroporation into asexual parasites of the 3D7 clone. Homologous recombination is expected to generate a pseudo- diploid locus in which neither of the two truncated copies encodes a functional kinase: the 5 ' copy lacks an essential glutamate residue in subdomain VIII and all downstream sequence including the 3'UTR; the 3' copy lacks the both the promoter region and the essential ATP orientation motif in subdomain I (Figure 4A).

Blasticidin-resistant parasite populations were obtained and shown by PCR analysis to contain parasites whose $p f e i k 1$ locus was disrupted. Clonal lines deriving from two independent transfection experiments were established by limiting dilution, and their genotypes were analysed by PCR (Figure 4B). The amplicon corresponding to the wild-type locus was not detected in clones $\mathrm{C} 1$ and $\mathrm{C} 8$ (lane 1), but was observed in wild-type parasites (lane 5). In contrast, PCR products that are diagnostic of both the 5' (lanes $3 \& 7$ ) and 3' (lanes $4 \& 8$ ) boundaries of the integrated plasmid were amplified from $\mathrm{C} 8$, but not 3D7 parasites (lanes 11 \& 12). The $\mathrm{C} 1$ and $\mathrm{C} 8$ clones also yielded a signal with primers that are specific for the transfection plasmid, and detect retained episomes or integrated concatemers. Integration was verified by Southern blot analysis of HindIII-digested genomic DNA (Figures 4C and 4D); the $12 \mathrm{~kb}$ band containing the wild-type locus is replaced in clones $\mathrm{C} 1$ and $\mathrm{C} 8$ by the expected two bands $(10.4 \mathrm{~kb}$ and $6.8 \mathrm{~kb}$ ) resulting from integration. The remaining 5.3 $\mathrm{kb}$ band is derived from linearized plasmid, or from digestion of concatemers of plasmid (which may or may not be integrated into the chromosome). These results confirm that the pfeik1 locus was indeed disrupted in clones C1 and $\mathrm{C} 8$, and demonstrate PfeIK1 is not required for completion of the asexual cycle in in vitro cultures. Additionally, asexual parasite cultures were synchronized and carefully monitored through several life cycles; samples were taken every 30 minutes and assessed for DNA content by flow cytometry [42]. No significant difference was observed in asexual cycle duration of the parental 3D7 
A
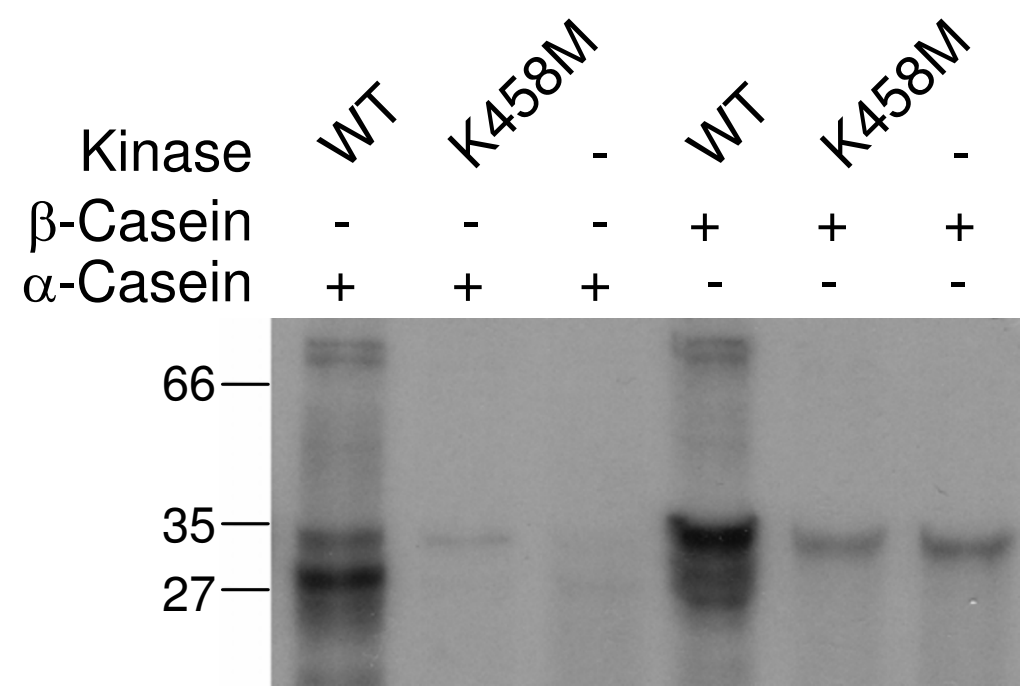

$\longleftarrow$ GST-PfelK1

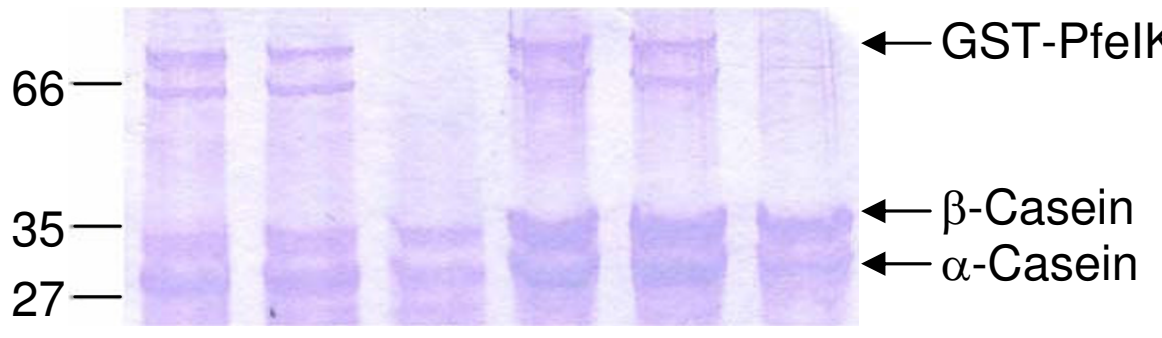

$\mathrm{B}$
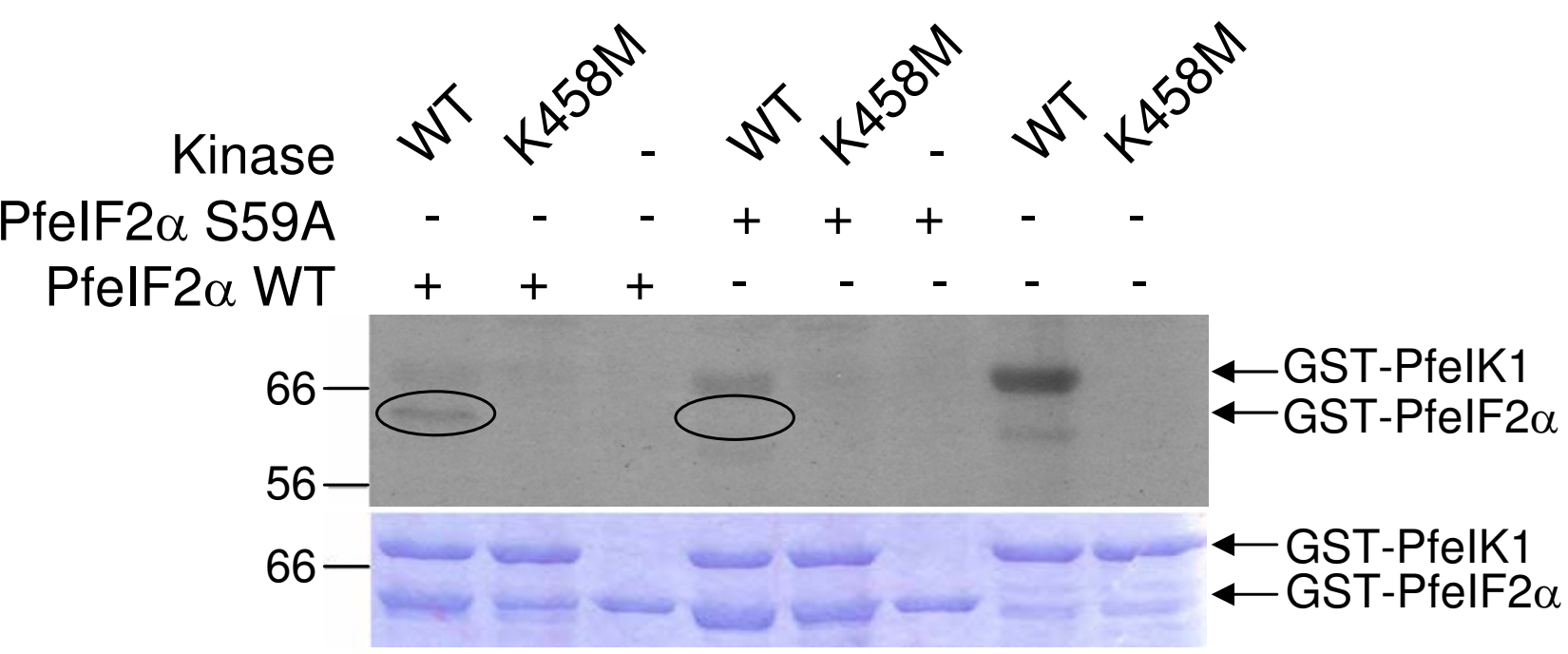

\section{Figure 3}

Kinase activity of PfelKI. A: GST-PfelKI phosphorylates the exogenous substrates $\alpha$-and $\beta$-casein. Kinase assays were performed using $10 \mu \mathrm{g} \alpha$-casein (left 3 lanes) or $\beta$-casein (right 3 lanes), in the presence of $2 \mu \mathrm{g}$ wild-type kinase catalytic domain (WT), catalytically inactive mutant (K458M) or no kinase (-). Upper panel: autoradiogram, lower panel: Coomassie blue-stained gel. B: GST-PfelKI autophosphorylates and can phosphorylate recombinant GST-PfelF2 $\alpha$, but not the mutant GST-PfelF2 $\alpha$ S59A. Kinase assays were performed using $2 \mu \mathrm{g}$ wild-type PfelK I catalytic domain (WT), or catalytically inactive mutant (K458M), or no kinase (-), in the presence of $10 \mu \mathrm{g}$ wild-type GST-PfelF2 $\alpha$ (left 3 lanes), targeted mutant GST-PfelF2 $\alpha$-S59A (middle 3 lanes) or no substrate (right 2 lanes). The position of the substrate is highlighted by ovals. 
A

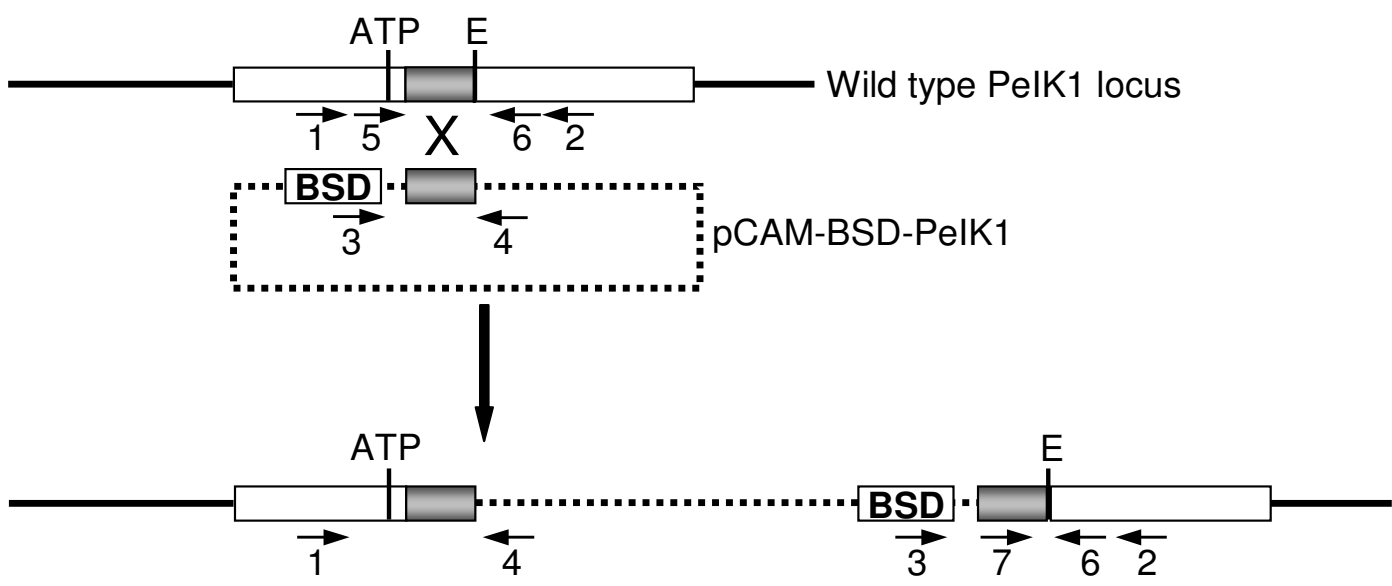

Recombined PelK1 locus

B

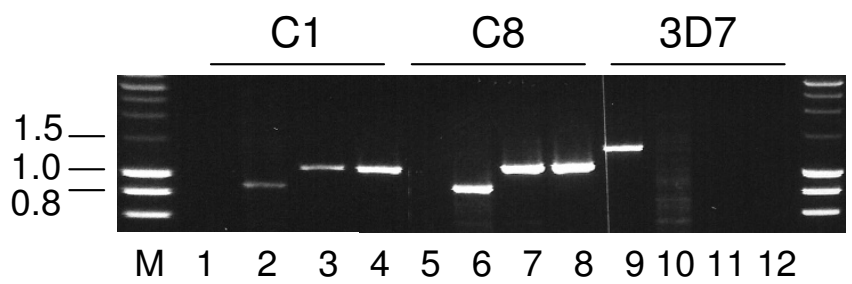

Lanes 1,5,9: 1+2 (1278bp)

Lanes 2,6,10: 3+4 (847bp)

Lanes 3,7,11: 1+4 (1050bp)

Lanes 4,8,12: 3+2 (1075bp)
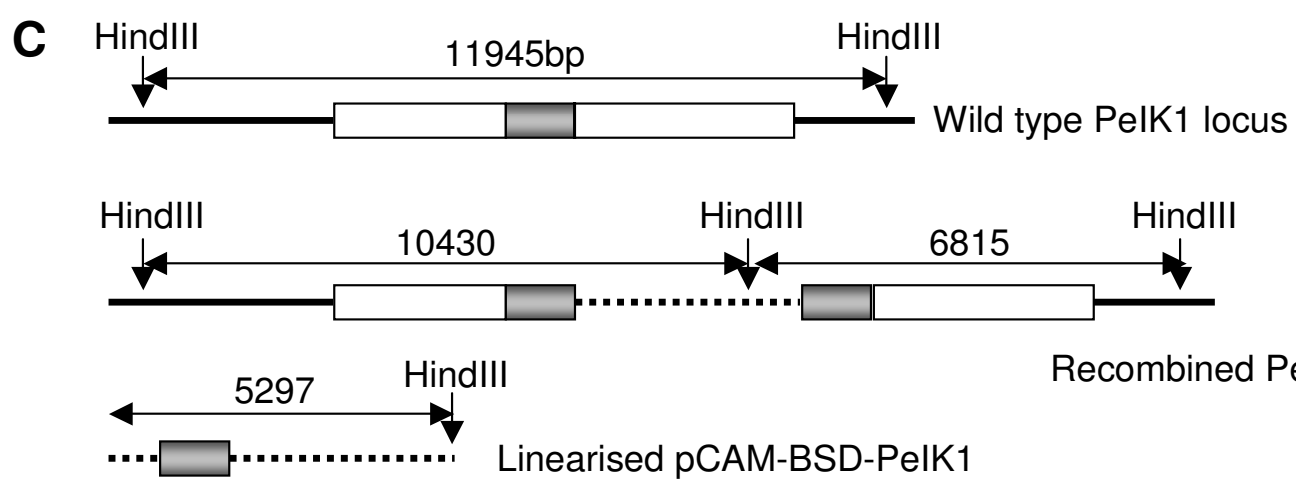

Recombined PelK1 locus

D $\quad 3 D 7$ C8
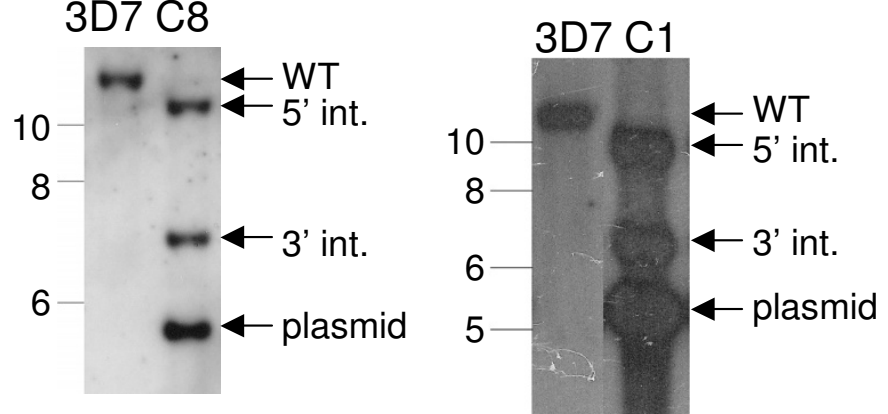

Figure 4 (see legend on next page) 
Figure 4 (see previous page)

Disruption of the pfeikl gene. A. Strategy for gene disruption. The transfection plasmid contains a PCR fragment spanning positions 1467-2255 of the entire $4.8 \mathrm{~kb}$ pfeik I coding sequence (as predicted on PlasmoDB). The fragment excludes two regions essential for catalytic activity, labelled 'ATP' (a glycine-rich region required for orientation of ATP) and 'E' (a glutamate residue required for structural stability of the enzyme). The positions of primers used for genotyping clones, and for nested PCR to genotype oocsyts are indicated by numbered arrows. B: PCR analysis. Genomic DNA isolated from pfeik 1 - clones $\mathrm{Cl}$ and C8, and from 3D7 wild-type parasites, was subjected to PCR using the indicated primers (see Figure 4A for primer locations). Lanes I, 5, 9: primers I + 2 (diagnostic for the wild-type locus); lanes 2, 6, I0: primers $3+4$ (diagnostic for the pCAMBSD-PfelKI plasmid); lanes 3, 7, I I: primers I + 4 (diagnostic for 5' integration boundary); lanes 4,8, 12: primers 3 + 2 (diagnostic for 3' integration boundary). $M=$ co-migrating markers. C: Schematic of expected sizes on Southern blot analysis of wildtype 3D7 parasites and pfeik I- parasites. D: Southern blot analysis of the pfeik l locus in wild-type 3D7 and pfeik I- clones Cl and C8. Genomic DNA was digested with HindIII, transferred to a Hybond membrane and probed with the pfeik I fragment that was used as the insert in the PCAM-BSD-PfelKI plasmid. Positions of the bands corresponding to the wild-type locus (WT), 5' integration (5' int.), 3' integration ( $3^{\prime}$ int.) and linearized plasmid (plasmid) are shown on the right. Sizes of co-migrating markers are indicated on the left.

clone and that of pfeik1- parasites; cycle times of $49.0 \mathrm{~h}+/$ -0.5 and $49.2 \mathrm{~h}+/-0.7$, respectively, were measured (Figure 5).

\section{elF2 $\alpha$ is not phosphorylated in pfeik I- clones during amino acid starvation}

To determine whether pfeik1- parasites were defective in responding to amino acid-limitation, we cultured these parasites in RPMI medium containing either all or no amino acids and assayed for eIF2 $\alpha$ phosphorylation through western blot analysis (Figure 6). We observed that pfeik $1^{-}$parasites were unable to modulate the phosphorylation state of eIF2 $\alpha$ in response to changing amino acid conditions, in direct contrast to wild-type parental clone $3 \mathrm{D} 7$. A further control was provided by performing the assay using a parasite clone lacking PfeIK2, another enzyme related to eIF2 $\alpha$ kinases (see Figure 2A; a full characterisation of PfeIK2 and pfeik2- parasite clones is to be published elsewhere). The pfeik2- parasites, which were generated using the same strategy as that described here for $p f e i k 1$ and were therefore also resistant to blasticidin, readily phosphorylated eIF2 $\alpha$ in amino acid starvation conditions, like wild-type 3D7 parasites. This demonstrates that the abolition of eIF2 $\alpha$ phosphorylation observed in pfeik1-parasites is not due to non-specific effects resulting from the genetic manipulations per-

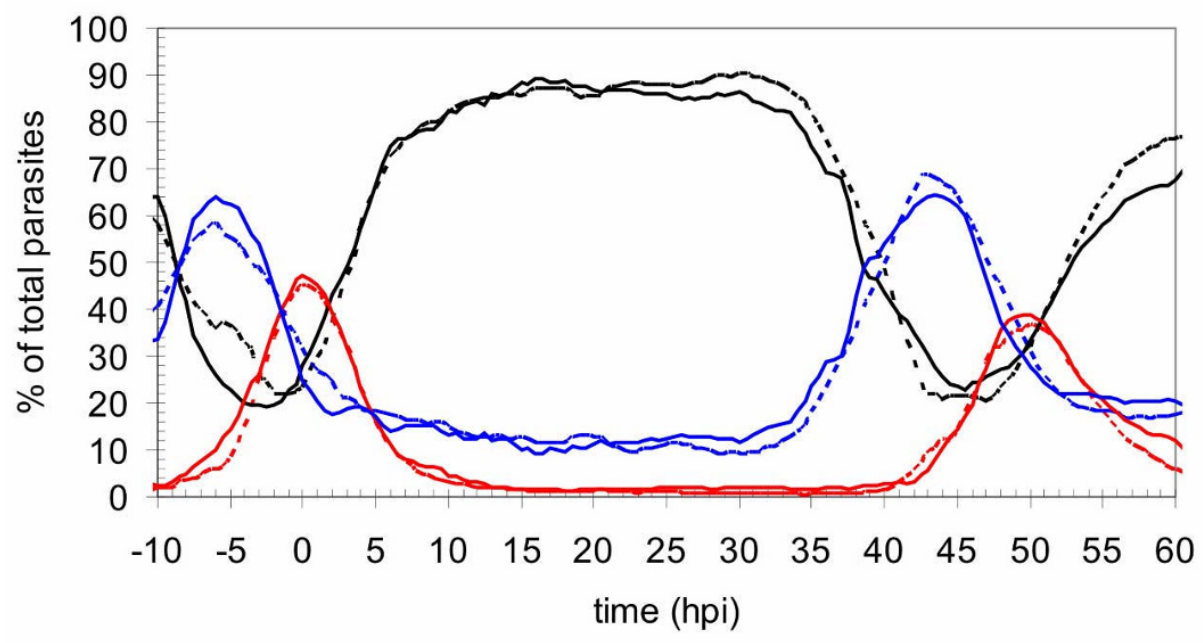

\section{Figure 5}

Disruption of the pfeikl gene does not affect asexual growth rate. Representative cycles of pfeikl-parasites and the parental 3D7 strain (dashed). Cycle points were semi-automatically collected fixed and stored at $4^{\circ} \mathrm{C}$ every 30 min over $\sim 4$ days. After permeabilization and RNAse treatment, the DNA content was analyzed by flow cytometry as previously described [42]. Mature schizonts $(\sim 16-32 \mathrm{~N})$, red line; S-phase $(\sim 2-8 \mathrm{~N})$, blue line; GI-phase (IN), black line. Percentage values as a function of time are shown; hpi: hours post-infection, referring to the mature schizont maxima as zero. 


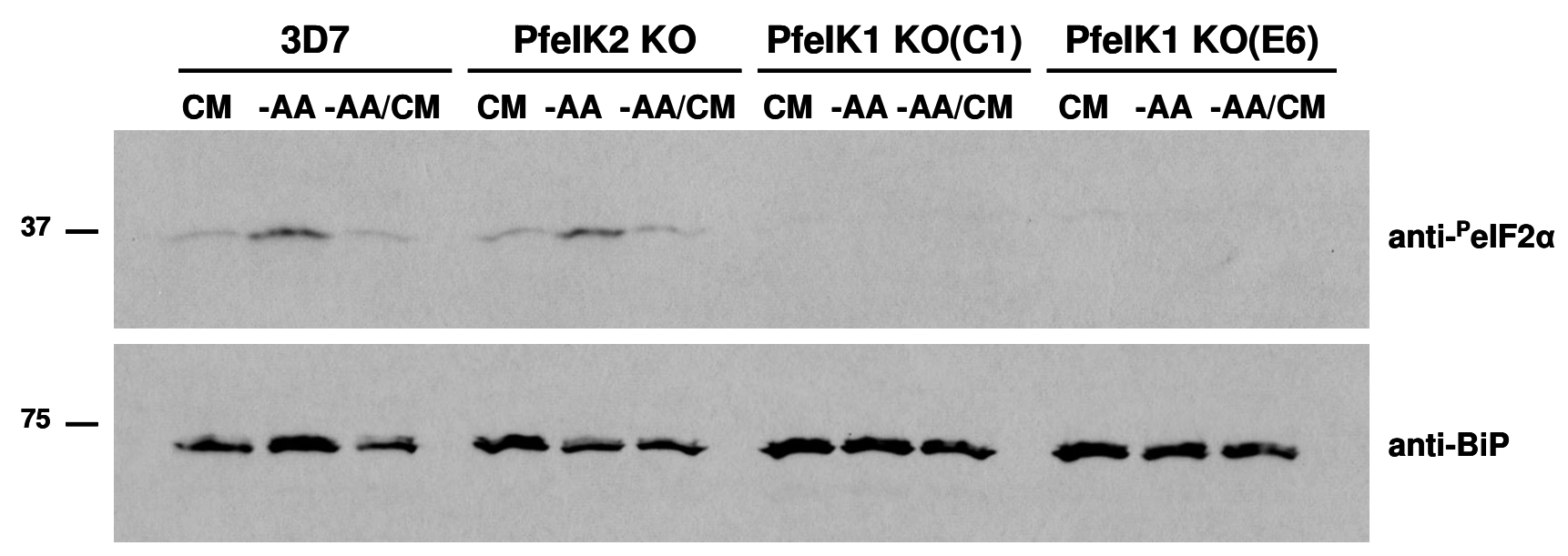

Figure 6

pfelK $I^{-}$parasites do not phosphorylate elF $2 \alpha$ in amino acid-limiting conditions. pfeik $I$ - clones $\mathrm{Cl}$ and $\mathrm{E} 6$, as well as the 3D7 parent clone and a pfeik2- clone used as controls, were synchronized to the late ring stage and equally partitioned into individual cultures. Growth of the parasites was continued up to 5 hours at $37^{\circ} \mathrm{C}$ in either complete RPMI (CM) or in RPMI lacking amino acids (-AA). CM was added back to one amino acid-deprived culture, and re-incubated for an additional 45 minutes. Total lysates from the parasites were prepared for SDS-PAGE, followed by immunoblotting with antibodies against phosphorylated elF $2 \alpha$ (anti-phosho elF $2 \alpha$ ). Antibodies against the endoplasmic reticulum marker BiP (anti-BiP) served as the loading control.

formed to obtain the mutant clones. Taken together, these data identify PfeIK1 as a crucial regulator of amino acid starvation stress response of intra-erythrocytic parasites.

\section{pfeikl-clones are competent for sexual development and mosquito infection}

The pfeik1- parasites were able to differentiate into gametocytes (data not shown). Further, qualitative results showed that $p f e i k 1$ - male gametocytes were competent to differentiate into gametes (in vitro exflagellation). To investigate whether PfeIK1 plays an essential role in subsequent life cycle stages, mosquitoes were fed with cultures of pfeik1- gametocytes. The numbers of oocysts associated with midguts dissected 10 days post-feeding, and the numbers of mosquitoes with sporozoite-positive salivary glands 16 days post-feeding, were then determined. This revealed that the complete sexual cycle can occur in the absence of PfeIK1, resulting in formation of oocysts and sporozoites (Table 1). Infection rates and median numbers of oocysts per infected mosquito are low relative to what is routinely observed in transmission experiments with the wild-type clone 3D7. However, this is to be expected from parasites that have been kept in continuous culture for a long period of time; in the present case it had taken $\sim 7$ months in culture to obtain knockout clones suitable for mosquito infection experiments. Circumstantial evidence that low infection levels are not a direct consequence of $p f e i k 1$ disruption is provided by the observation that our control for these experiments (mock-transfected 3D7 that had been cultured for the same duration, in parallel to the pfeik1- parasites), had completely lost the ability to produce gametocytes and therefore infect mosquitoes. Importantly, to verify that the parasites infecting the mosquitoes had not reverted to the wild-type genotype, midguts from infected mosquitoes were collected 10 days post-feeding, from which total DNA was extracted and used in nested PCR experiments. The wild-type locus could be amplified from mosquitoes infected with wild-type 3D7 parasites, but not from those infected with pfeik1- C8 parasites (Figure 7, lower panel, lanes 1, 3, 5). Conversely, the amplicon diagnostic of the 3 ' boundary of the integrated plasmid could only be amplified from midguts of pfeik1- C8-infected mosquitoes, but not from mosquitoes infected with wild-type parasites (lanes 2, 4, 8).

On the basis of the similarities between PfeIK1 and GCN2, we hypothesized that PfeIK1 is involved in modulating the response to amino acid starvation depicted in Figure $1 \mathrm{~B}$. That this is indeed the case was demonstrated through a reverse genetics approach: parasites lacking PfeIK1 do not phosphorylate eIF2 $\alpha$ in response to aminoacid depletion (Figure 6). Future work will determine the impact of PfeIK1 activation on both the rate of translation and the possible selection of specific messages that are translated under stress conditions. Overall, the data presented here suggest that eIF $2 \alpha$ phosphorylation in response to amino-acid starvation is not essential to parasite survival during the erythrocytic asexual cycle (at least 


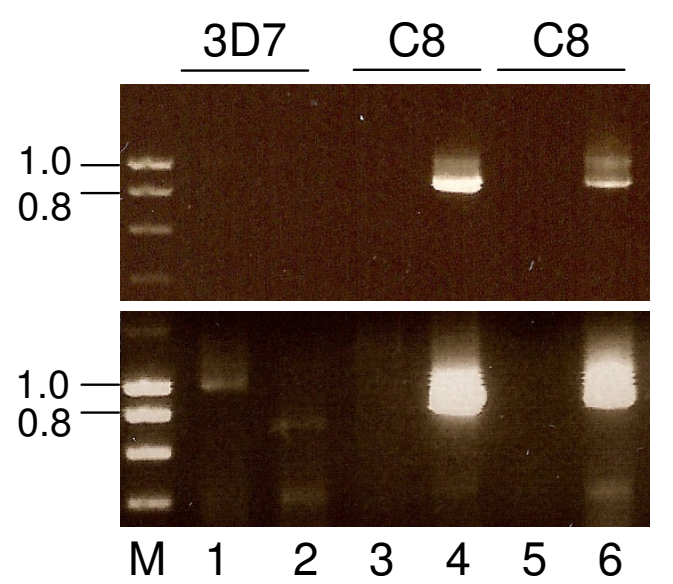

\section{Figure 7}

Analysis of the parasite genotypes in mosquito infections. Genomic DNA extracted from a wild-type 3D7infected mosquito and from two mosquitoes infected with clone $\mathrm{C} 8$ was analysed by nested PCR; primer positions are indicated in Fig. 3A. The inner PCR product is shown. Lanes I, $3 \& 5$ are diagnostic for the wild-type locus (primers I +2 , followed by $5+6$ ). Lanes $2,4 \& 6$ are diagnostic for the 3 ' boundary of plasmid integration (primers $3+2$, followed by $7+6)$. The 3D7 infected mosquito used here serves as a control for PCR amplification of the wild-type locus from a midgut, but came from a separate experiment and hence did not provide a control for infection prevalence or intensity. Upper panel: shorter exposure; lower panel: longer exposure to reveal the wild-type band in lane $I$ and its absence in the $\mathrm{C} 8$ samples. $M=$ co-migrating markers.

in an in vitro cultivation context), or for completion of sporogony.

Commitment to gametocytogenesis has been proposed to be linked to stress response, and eIF2 $\alpha$ might possibly be involved in this process. At first sight, the data presented here suggest that PfeIK1 does not regulate gametocytogenesis, since $p f e i k 1^{-}$parasite are able to undergo sexual development. However, caution must be exercised, as compensatory mechanisms can be at play in knock-out parasites. Indeed, in a similar situation concerning another protein kinase family, it was observed that disruption of the gene encoding one of the two P. falciparum mitogen-activated protein kinases (MAPKs), pfmap-1, does not cause any detectable phenotype, but that pfmap1- parasites overexpress the second parasite MAPK, Pfmap2 [40]. A similar compensation mechanism may operate between the three PfeIKs represented in the parasite kinome (Figure 2A). Even though compensatory mechanisms to permit sexual differentiation are presumably less likely to occur than those allowing the survival of asexual parasites (because of the absence of a true selection pressure), it cannot be formally excluded that PfeIK1 plays a role in gametocytogenesis in a wild-type parasite background. Investigating this possibility will require inducible and/or multiple knock-outs and the availability of mono-specific antibodies to monitor the levels of each PfeIK in parasites lacking one of them.

\section{Conclusion}

Phylogenetic analysis indicates that the $P$. falciparum kinome includes three putative eIF $2 \alpha$ kinases. One of these, PfPK4, was previously shown to phosphorylate a peptide corresponding to the target region of human eIF $2 \alpha$ [27]. It is demonstrated here that PfeIK1 is able to phosphorylate the conserved regulatory site on the Plasmodium orthologue of the translation factor in vitro, and that eIF $2 \alpha$ phopshorylation in response to amino-acid starvation does not occur in pfeik 1 - parasites. The present study thus establishes that malaria parasites possess the molecular machinery that pertains to stress-dependent regulation of translation, and that this machinery is actually used in stress response.

\section{Competing interests}

The authors declare that they have no competing interests.

\section{Authors' contributions}

CF carried out molecular cloning, kinase assays, parasite genetic manipulations and analysis, participated in bioinformatic analysis and drafted the manuscript. SB carried out parasite starvation and immunoblotting experiments. IR analysed parasite growth. JW participated in sequence alignments and generated the phylogenetic tree. LRC car-

Table I: Oocyst and sporozoite formation by pfeik I-parasite clones.

\begin{tabular}{lllll}
\hline Clone & Exp. no & $\begin{array}{l}\text { \% Infection } \\
\text { (no. infected/no. dissected) }\end{array}$ & $\begin{array}{l}\text { Median oocyst no. per infected } \\
\text { mosquito (range) }\end{array}$ & $\begin{array}{l}\text { \% Sporozoite-positive } \\
\text { (no. infected/no. dissected) }\end{array}$ \\
\hline $\mathrm{Cl}$ & 1 & $15 \%(2 / 14)$ & $1.5(I-2)$ & $\mathrm{ND}$ \\
\hline $\mathrm{C} 8$ & 1 & $44 \%(7 / 16)$ & $10(1-34)$ & $37 \%(7 / 19)$ \\
\hline $\mathrm{C} 8$ & 2 & $20 \%(5 / 25)$ & $2(1-5)$ & $\mathrm{ND}$ \\
\hline
\end{tabular}

There was no difference in the prevalence of mosquitoes positive for the oocyst and sporozoites stages (exp. 2 , $\mathrm{P}=0.74$ ). 
ried out mosquito infections and participated in their analysis. DEG participated in conception of the study. CD conceived of the study, participated in its design and coordination and helped to draft the manuscript. All authors read and approved the final manuscript.

\section{Acknowledgements}

This work is based on gene identification made possible by the availability of the genome sequences of $P$. falciparum and $P$. berghei, and of the PlasmoDB database. Financial support for the Plasmodium Genome Consortium was provided by the Burroughs Wellcome Fund, the Wellcome Trust, the National Institutes of Health (NIAID) and the U.S. Department of Defense, Military Infectious Diseases Research Program. Financial Support for PlasmoDB was provided by the Burroughs Wellcome Fund. We thank Luc Reininger for his input at the onset of this project and for frequent discussions about this and other topics, and Jacques Chevalier (Service Scientifique de I'Ambassade de France in London) for continuing support. Work in the C.D. laboratory is funded by Inserm, the FP6 (SIGMAL and ANTIMAL projects, and BioMalPar Network of Excellence) and FP7 (MALSIG project) programmes of the European Commission and a grant from the Novartis Institute for Tropical Diseases. C.F is the recipient of a PhD studentship awarded by the Wellcome Trust.

\section{References}

I. Bozdech Z, Llinas M, Pulliam BL, Wong ED, Zhu J, DeRisi JL: The transcriptome of the intraerythrocytic developmental cycle of Plasmodium falciparum. PLoS Biol 2003, I:E5.

2. Coulson RM, Hall N, Ouzounis CA: Comparative genomics of transcriptional control in the human malaria parasite Plasmodium falciparum. Genome Res 2004, I 4: I548-1554.

3. De Silva EK, Gehrke AR, Olszewski K, Leon I, Chahal JS, Bulyk ML, Llinas M: Specific DNA-binding by apicomplexan AP2 transcription factors. Proc Natl Acad Sci USA 2008, 105:8393-8398.

4. Hall N, Karras M, Raine JD, Carlton JM, Kooij TW, Berriman M, Florens L, Janssen CS, Pain A, Christophides GK, James K, Rutherford K, Harris B, Harris D, Churcher C, Quail MA, Ormond D, Doggett J, Trueman HE, Mendoza J, Bidwell SL, Rajandream MA, Carucci DJ, Yates JR 3rd, Kafatos FC, Janse C], Barrell B, Turner CM, Waters AP, Sinden RE: A comprehensive survey of the Plasmodium life cycle by genomic, transcriptomic, and proteomic analyses. Science 2005, 307:82-86.

5. Paton MG, Barker GC, Matsuoka H, Ramesar J, Janse CJ, Waters AP, Sinden RE: Structure and expression of a post-transcriptionally regulated malaria gene encoding a surface protein from the sexual stages of Plasmodium berghei. Mol Biochem Parasitol 1993, 59:263-275.

6. Mair GR, Braks JA, Garver LS, Wiegant JC, Hall N, Dirks RW, Khan SM, Dimopoulos G, Janse CJ, Waters AP: Regulation of sexual development of Plasmodium by translational repression. Science 2006, 3 I 3:667-669.

7. Engelberg D: Stress-activated protein kinases-tumor suppressors or tumor initiators? Semin Cancer Biol 2004, 14:27 I-282.

8. Dorin D, Semblat JP, Poullet P, Alano P, Goldring JP, Whittle C, Patterson S, Chakrabarti D, Doerig C: PfPK7, an atypical MEKrelated protein kinase, reflects the absence of classical threecomponent MAPK pathways in the human malaria parasite Plasmodium falciparum. Mol Microbiol 2005, 55:184-196.

9. Dorin D, Alano P, Boccaccio I, Ciceron L, Doerig C, Sulpice R, Parzy $D$ : An atypical mitogen-activated protein kinase (MAPK) homologue expressed in gametocytes of the human malaria parasite Plasmodium falciparum. Identification of a MAPK signature. J Biol Chem 1999, 274:299/2-29920.

10. Ward P, Equinet L, Packer J, Doerig C: Protein kinases of the human malaria parasite Plasmodium falciparum: the kinome of a divergent eukaryote. BMC Genomics 2004, 5:79.

II. Sullivan WJ Jr, Narasimhan J, Bhatti MM, Wek RC: Parasite-specific elF2 (eukaryotic initiation factor-2) kinase required for stress-induced translation control. Biochem J 2004, 380:523-531.
12. Wek RC, jiang HY, Anthony TG: Coping with stress: elF2 kinases and translational control. Biochem Soc Trans 2006, 34:7-II.

13. Holcik M, Sonenberg N: Translational control in stress and apoptosis. Nat Rev Mol Cell Biol 2005, 6:3 I 8-327.

14. Proud CG: elF2 and the control of cell physiology. Semin Cell Dev Biol 2005, 16:3-12.

15. Choi SY, Scherer BJ, Schnier J, Davies MV, Kaufman RJ, Hershey JW: Stimulation of protein synthesis in COS cells transfected with variants of the alpha-subunit of initiation factor elF-2. J Biol Chem 1992, 267:286-293.

16. Murtha-Riel P, Davies MV, Scherer BJ, Choi SY, Hershey JW, Kaufman RJ: Expression of a phosphorylation-resistant eukaryotic initiation factor 2 alpha-subunit mitigates heat shock inhibition of protein synthesis. J Biol Chem 1993, 268: I2946-I295I.

17. Colthurst DR, Campbell DG, Proud CG: Structure and regulation of eukaryotic initiation factor elF-2. Sequence of the site in the alpha subunit phosphorylated by the haem-controlled repressor and by the double-stranded RNA-activated inhibitor. Eur J Biochem 1987, 166:357-363.

18. Wek SA, Zhu S, Wek RC: The histidyl-tRNA synthetase-related sequence in the elF-2 alpha protein kinase GCN2 interacts with tRNA and is required for activation in response to starvation for different amino acids. Mol Cell Biol 1995, I5:4497-4506.

19. Chen JJ, London IM: Regulation of protein synthesis by hemeregulated elF-2 alpha kinase. Trends Biochem Sci 1995, 20:105-108.

20. Sudhakar A, Ramachandran A, Ghosh S, Hasnain SE, Kaufman RJ, Ramaiah KV: Phosphorylation of serine $5 \mathrm{I}$ in initiation factor 2 alpha (elF2 alpha) promotes complex formation between elF2 alpha(P) and eIF2B and causes inhibition in the guanine nucleotide exchange activity of elF2B. Biochemistry 2000, 39:12929-12938.

21. Miranda-Saavedra D, Stark MJ, Packer JC, Vivares CP, Doerig C, Barton $\mathrm{GJ}$ : The complement of protein kinases of the microsporidium Encephalitozoon cuniculi in relation to those of Saccharomyces cerevisiae and Schizosaccharomyces pombe. BMC Genomics 2007, 8:309.

22. Mohrle JJ, Zhao Y, Wernli B, Franklin RM, Kappes B: Molecular cloning, characterization and localization of PfPK4, an elF2alpha kinase-related enzyme from the malarial parasite Plasmodium falciparum. Biochem J 1997, 328(Pt 2):677-687.

23. Anamika, Srinivasan N, Krupa A: A genomic perspective of protein kinases in Plasmodium falciparum. Proteins 2005, 58:180-189.

24. Huson DH, Bryant D: Application of phylogenetic networks in evolutionary studies. Mol Biol Evol 2006, 23:254-267.

25. Ho SN, Hunt HD, Horton RM, Pullen JK, Pease LR: Site-directed mutagenesis by overlap extension using the polymerase chain reaction. Gene 1989, 77:51-59.

26. Sidhu $A B$, Valderramos SG, Fidock DA: pfmdrI mutations contribute to quinine resistance and enhance mefloquine and artemisinin sensitivity in Plasmodium falciparum. Mol Microbiol 2005, 57:913-926.

27. Lin DT, Goldman ND, Syin C: Stage-specific expression of a Plasmodium falciparum protein related to the eukaryotic mitogen-activated protein kinases. Mol Biochem Parasitol 1996, 78:67-77.

28. Russo I, Oksman A, Vaupel B, Goldberg DE: A calpain unique to alveolates is essential in P. falciparum and its knockdown reveals an involvement in pre-S-phase development. Proc Natl Acad Sci USA 106:1554-1559.

29. Carter R, Ranford-Cartwright L, Alano P: The culture and preparation of gametocytes of Plasmodium falciparum for immunochemical, molecular, and mosquito infectivity studies. Methods Mol Biol 1993, 21 :67-88.

30. Ranford-Cartwright LC, Balfe P, Carter R, Walliker D: Frequency of cross-fertilization in the human malaria parasite Plasmodium falciparum. Parasitology 1993, I07(Pt I): I I-I8.

31. Dar AC, Dever TE, Sicheri F: Higher-order substrate recognition of elF2alpha by the RNA-dependent protein kinase PKR. Cell 2005, I 22:887-900.

32. Bahl A, Brunk B, Crabtree J, Fraunholz MJ, Gajria B, Grant GR, Ginsburg H, Gupta D, Kissinger JC, Labo P, Labo P, Li L, Mailman MD, Milgram AJ, Pearson DS, Roos DS, Schug J, Stoeckert CJ Jr, Whetzel P: the Plasmodium PlasmoDB: genome resource. A database 
integrating experimental and computational data. Nucleic Acids Res 2003, 31:212-215.

33. Hanks SK: Genomic analysis of the eukaryotic protein kinase superfamily: a perspective. Genome Biol 2003, 4: III.

34. Mathews MB, Sonenberg N, Hershey JWB: Translational Control in Biology and Medicine. Cold Spring Harbor Laboratory Press; NY, USA; 2007.

35. Dever TE: Gene-specific regulation by general translation factors. Cell 2002, I08:545-556.

36. Romano PR, Garcia-Barrio MT, Zhang X, Wang Q, Taylor DR, Zhang F, Herring C, Mathews MB, Qin J, Hinnebusch AG: Autophosphorylation in the activation loop is required for full kinase activity in vivo of human and yeast eukaryotic initiation factor 2alpha kinases PKR and GCN2. Mol Cell Biol 1998, 18:2282-2297.

37. Padyana AK, Qiu H, Roll-Mecak A, Hinnebusch AG, Burley SK: Structural basis for autoinhibition and mutational activation of eukaryotic initiation factor 2alpha protein kinase GCN2. J Biol Chem 2005, 280:29289-29299.

38. Garcia MA, Meurs EF, Esteban M: The dsRNA protein kinase PKR: virus and cell control. Biochimie 2007, 89:799-8I I.

39. Le Roch KG, Zhou Y, Blair PL, Grainger M, Moch JK, Haynes JD, De La Vega P, Holder AA, Batalov S, Carucci DJ, Winzeler EA: Discovery of gene function by expression profiling of the malaria parasite life cycle. Science 2003, 30 I: I503-1508.

40. Dorin-Semblat D, Quashie N, Halbert J, Sicard A, Doerig C, Peat E, Ranford-Cartwright L, Doerig C: Functional characterization of both MAP kinases of the human malaria parasite Plasmodium falciparum by reverse genetics. Mol Microbiol 2007, 65: $1170-1180$.

41. Dorin-Semblat D, Sicard A, Doerig C, Ranford-Cartwright L, Doerig C: Disruption of the PfPK7 gene impairs schizogony and sporogony in the human malaria parasite Plasmodium falciparum. Eukaryot Cell 2008, 7:279-285.

42. Liu J, Gluzman IY, Drew ME, Goldberg DE: The role of Plasmodium falciparum food vacuole plasmepsins. J Biol Chem 2005, 280: 1432-1437.

Publish with Bio Med Central and every scientist can read your work free of charge

"BioMed Central will be the most significant development for disseminating the results of biomedical research in our lifetime. "

Sir Paul Nurse, Cancer Research UK

Your research papers will be:

- available free of charge to the entire biomedical community

- peer reviewed and published immediately upon acceptance

- cited in PubMed and archived on PubMed Central

- yours - you keep the copyright

Submit your manuscript here:

http://www.biomedcentral.com/info/publishing_adv.asp
BioMedcentral 